\title{
Emerging ceftazidime-avibactam resistance against carbapenem resistant Escherichia coli and Klebsiella pneumoniae in Lebanon
}

\author{
Ghena M Sobh', AbdulKarim M El Karaaoui ${ }^{1}$, \\ Mira El Chaar ${ }^{2}$, George F Araj ${ }^{1}$
}

\section{Abstract}

Introduction: Ceftazidime-avibactam (CZA) has been introduced as a novel therapy to essentially combat the rising trends of carbapenem resistant Enterobacteriaceae. In the absence of in vitro data about the activity of this drug against carbapenem resistant (CR) Escherichia coli and Klebsiella pneumoniae in Lebanon, this study was warranted.

Method: A total of 150 isolates, identified using the MALDI-TOF, encompassing 50 CR E. coli, 60 CR K. pneumoniae, and 10 isolates each of extended-spectrum Beta-lactamases (ESBLs), and non-CR multidrug-resistant (MDR) of each species were analyzed. The minimum inhibitory concentration (MIC) for CZA was determined by the E-test (Liofilchem, Roseto degliAbruzzi, Italy). In addition, the disk diffusion (DD) test was used to determine the activity of CZA and of the antimicrobials routinely used to test for such pathogens.

Results: The CZA activity against the 50 CR E. coli showed an $\mathrm{MIC}_{50} \geq 256 \mu \mathrm{g} / \mathrm{mL}, \mathrm{MIC}_{90} \geq 256 \mu \mathrm{g} / \mathrm{mL}$, and an $\mathrm{MIC}$ range of 0.023 to $\geq 256 \mu \mathrm{g} / \mathrm{mL}$, reflecting a susceptibility of $40 \%$. As For the $60 \mathrm{CR} \mathrm{K}$. pneumoniae isolates, the $\mathrm{MIC}_{50}$ was $\geq 256 \mu \mathrm{g} / \mathrm{mL}$, $\mathrm{MIC}_{90} \geq 256 \mu \mathrm{g} / \mathrm{mL}$, and the MIC range was 0.094 to $\geq 256 \mu \mathrm{g} /$ $\mathrm{mL}$, reflecting a susceptibility of $35 \%$. However, uniform CZA susceptibility (100\%) was detected against ESBL and MDR isolates of both species, being comparable or higher to the routinely used antimicrobials.
1 Department of Pathology and Laboratory Medicine, American University of Beirut, Medical Center, Beirut, Lebanon.

2 Faculty of Health Sciences, University of Balamand, Beirut, Lebanon.

Contact information:

George F. Araj, PhD, D (ABMM), FAAM.

Address: Professor and Director of Clinical Microbiology Division. Department of Pathology \& Laboratory Medicine, American University of Beirut Medical

”garaj@aub.edu.lb 
Conclusion: Although CZA was recently introduced into Lebanon, it was surprising to note this low activity of CZA against CR E. coli and CR K. pneumoniae. To explain such findings, it is worth pursuing investigations related to antimicrobial utilization in clinical practice and antimicrobial stewardship. Moreover, genotypic determination is needed to be revealed to help explain the observed phenotypic resistance.

\section{Keywords}

Antimicrobial Resistance; Ceftazidime Avibactam; Carbapenem Resistance, CR E. Coli, CR K. Pneumoniae; Lebanon.

\section{Introduction}

The surging encounter of resistant Gram-negative bacteria is imposing a great global public threat, as well as increased mortality, morbidity, hospital stay, and not to forget the economic burden [1]. This is essentially attributed to the increasing rates of ESBL, failure in their treatment which led to subsequent increase in $C R E$, and the deprivation of effective antimicrobial agents to treat such highly resistant isolates [2] [https://www.who.int/news/ item/27-02-2017-who-publishes-list-of-bacteria-forwhich-new-antibiotics-are-urgently-needed].

Ceftazidime-avibactam has been introduced as one of the therapeutic options to treat such resistant isolates. It received FDA approval for the treatment of complicated urinary tract infections including pyelonephritis, complicated intra-abdominal infections and hospital acquired pneumonia/ ventilator associated pneumonia [3, 4]. This drug is a novel antibiotic combination consisting of a third-generation cephalosporin (ceftazidime) combined with a non- $\beta$-lactam $\beta$-lactamase inhibitor (avibactam). The latter is a synthetic non- $\beta$-Lactam molecule that protects $\beta$-lactams from Extended spectrum $\beta$-lactamases and carbapenem resistant Gram-negative bacteria by inhibiting Class A, C and some Class D $\beta$-lactamases enzymes, by covalently acetylating the $\beta$-lactamases targets mainly the serine domain, but not the metallo $\beta$-lactamases [5].

Although ceftazidime-avibactam is relatively new in the market, bacterial resistance to this drug has been reported worldwide, especially among Pseudomonas spp., Acinetobacter spp. and carbapenem resistant Enterobacteriaceae (CRE) isolates [6]. In Lebanon, ceftazidime-avibactam has been available for a few years now. However, no data is available about the prevalence of its resistance, thus prompted us to assess the in vitro activity of CZA against multi-resistant $E$. coli and K. pneumoniae pathogens in this country.

\section{Method}

\section{Bacterial isolates and their identification}

Non-duplicate isolates consisting of 70 E. coli (10 ESBLs, $10 \mathrm{MDR}$, and 50 (RE isolates) and $80 \mathrm{~K}$. pneumoniae (10 ESBLs, $10 \mathrm{MDR}$, and $60 \mathrm{CRE}$ isolates) recovered from different clinical specimens that were submitted for investigation at the Clinical Microbiology Laboratory, Department of Pathology and Laboratory Medicine, American University of Beirut Medical Center (AUBMC) during the period 
between May 2019 and March 2021. Identification of the isolates was done using the matrix-assisted laser desorption/ionization time of flight (MALDITOF) system (Bruker Daltonik, GmbH, Bremen, Germany).

\section{Antimicrobial susceptibility testing}

Antimicrobial susceptibility testing was performed using the E-test for minimal inhibitory concentration (MIC) determination and the disk diffusion (DD) test as reported in a previous study done at our center [7]. Both the CZA MIC strips (concentration range $\leq 0.016$ and $\geq 256 \mu \mathrm{g} / \mathrm{mL}$ ) and the CZA disks (50 $\mu \mathrm{g})$ were obtained from Liofilchem, Scozia, Italy. The 2020 Clinical and Laboratory Standards Institute (CLSI) CZA MICs' breakpoints $(\mu \mathrm{g} / \mathrm{mL})$ were used to interpret the CZA susceptibility category for Enterobacteriaceae as susceptible $(\leq 8 \mu \mathrm{g} / \mathrm{mL})$ and resistant $(\geq 16 \mu \mathrm{g} / \mathrm{mL})$, and for the CZA (50 $\mu \mathrm{g})$ $\mathrm{DD}$, the susceptible and resistant zone of inhibition $(\mathrm{mm})$, were $\geq 21$, and $\leq 20$, respectively. The other antimicrobial agents tested by DD are the ones routinely used for testing these pathogens, and their results were also interpreted according to the 2020 CLSI guidelines.

The categorization of bacterial resistance to antimicrobial agents was based on the definition created by a group of international experts initiated by the European Center for Disease Prevention and Control and the Centers for Disease Control and Prevention. They defined MDR as acquired nonsusceptibility to at least one agent in three or more antimicrobial categories [8]. In our study, resistance to cefoxitin was also included in categorizing $E$. coli and $K$. pneumoniae isolates as MDR. The characterization of $E$. coli and $K$. pneumoniae isolates as ESBL producers was carried out as previously reported in a study done at our center [9]. Carbapenem resistance was determined based on resistance to one of the carbapenem agents: ertapenem, imipenem, or meropenem.

\section{Quality Control}

The quality of testing with E-test and DD test was ensured using the American Type Culture Collection (ATCC) quality control strain of E. coli (ATCC 25922).

\section{Results}

The clinical sources for the $C R$ isolates of $E$. coli and $K$. pneumoniae, respectively, were urinary $34 \%$ and 23\%; DTA 8\% and 25\%; skin screenings $16 \%$ and $18 \%$; blood $22 \%$ and $20 \%$. Other sources with very low isolate recovery (20\% and $14 \%$ ) included tissues, fluids, catheters, and wounds.

The distribution of the tested isolates according to their CZA MICs range, $\mathrm{MIC}_{50}$, and $\mathrm{MIC}_{90}$ among the ESBL, MDR and CR E. coli, and K. pneumoniae is presented in Table 1.

The CZA MICs activity against the 50 CR E. coli reflected a susceptibility of $40 \%$, while the CZA activity against the $60 \mathrm{CR} K$. pneumoniae isolates showed a susceptibility of 35\%. However, uniform CZA susceptibility (100\%) was detected against the ESBL and MDR isolates of both species, being comparable and higher to the routinely used antimicrobials.

Figure 1 shows the scatter of CZA MICs for both $\mathrm{CR}$ isolates of $E$. coli and $K$. pneumoniae, ranging between 0.016 and $\geq 256 \mu \mathrm{g} / \mathrm{mL}$.

The disk diffusion results of the different bacterial types of resistance against CZA and other routinely tested antimicrobial agents used for these pathogens in a clinical setting are shown in Table 2.

Although CZA susceptibility rates were lower than those shown for aminoglycosides for $E$. coli and close to those shown for these drugs in $K$. pneumoniae, they were higher than those reported for the other tested antimicrobial agents for both species.

Comparing the DD results with those of the MICs results, no discrepant findings were shown among the tested CR K. pneumoniae isolates. However, among the tested CR E. coli isolates, 2 discrepancies 
Table 1. Minimum inhibitory concentration ( $\mathrm{MIC} \mu \mathrm{g} / \mathrm{mL}$ ) of resistant $E$. coli and $K$. pneumoniae isolates against CZA and carbapenems.

\begin{tabular}{|c|c|c|c|c|c|c|}
\hline \multicolumn{7}{|c|}{ MICs $(\mu \mathrm{g} / \mathrm{mL})$ results against resistant $E$. coli and $K$. pneumoniae } \\
\hline Antimicrobial & Organism & $\begin{array}{c}\text { Resistance } \\
\text { Characteristic }\end{array}$ & MIC 50 & MIC 90 & Range & $\begin{array}{c}\% \text { Susceptible in } \\
\text { Category }\end{array}$ \\
\hline \multirow{6}{*}{ CZA } & \multirow{3}{*}{ E. coli } & $\operatorname{ESBL}(n=10)$ & 0.19 & 0.5 & $0.094-0.75$ & 100 \\
\hline & & $\operatorname{MDR}(n=10)$ & 0.38 & 2 & $0.125-3$ & 100 \\
\hline & & $C R(n=50)$ & $\geq 256$ & $\geq 256$ & $0.023-\geq 256$ & 40 \\
\hline & \multirow{3}{*}{ K. pneumoniae } & ESBL $(n=10)$ & 0.38 & 1.5 & $0.25-3$ & 100 \\
\hline & & $\operatorname{MDR}(n=10)$ & 0.5 & 2 & $0.25-2$ & 100 \\
\hline & & $C R(n=60)$ & $\geq 256$ & $\geq 256$ & $0.094-\geq 256$ & 35 \\
\hline \multirow{2}{*}{ ERT } & E. coli & $C R(n=50)$ & $\geq 32$ & $\geq 32$ & $2-\geq 32$ & 0 \\
\hline & K. pneumoniae & $C R(n=60)$ & $\geq 32$ & $\geq 32$ & $1.5-\geq 32$ & 0 \\
\hline \multirow{2}{*}{ IMP } & E. coli & $C R(n=50)$ & $\geq 32$ & $\geq 32$ & $0.064-\geq 32$ & 10 \\
\hline & K. pneumoniae & $C R(n=60)$ & $\geq 32$ & $\geq 32$ & $0.25-\geq 32$ & 7 \\
\hline \multirow{2}{*}{ MERO } & E. coli & $C R(n=50)$ & $\geq 32$ & $\geq 32$ & $0.25-\geq 32$ & 8 \\
\hline & K. pneumoniae & $C R(n=60)$ & $\geq 32$ & $\geq 32$ & $0.25-\geq 32$ & 8 \\
\hline
\end{tabular}

$\mathrm{CR}=$ Carbapenem resistant, CZA: Ceftazidime-Avibactam, ERT: Ertapenem, IMP: Imipenem, MERO: Meropenem.

Table 2. Disk diffusion susceptibility of resistant $E$. coli and $K$. pneumoniae isolates to CZA and other antimicrobial agents.

\begin{tabular}{|c|c|c|c|c|c|c|}
\hline \multicolumn{7}{|c|}{ Percent (\%) susceptibility among } \\
\hline \multirow{3}{*}{$\begin{array}{l}\text { Antimicrobial } \\
\text { agents }\end{array}$} & \multicolumn{3}{|c|}{ E. coli } & \multicolumn{3}{|c|}{ K. pneumoniae } \\
\hline & ESBL & MDR & CRE & ESBL & MDR & CRE \\
\hline & \multicolumn{2}{|c|}{$n=10$} & $n=50$ & \multicolumn{2}{|c|}{$n=10$} & $n=60$ \\
\hline $\begin{array}{l}\text { Ceftazidime- } \\
\text { Avibactam* }\end{array}$ & 100 & 100 & 32 & 100 & 100 & 35 \\
\hline Amikacin & 100 & 100 & 86 & 100 & 100 & 40 \\
\hline Ciprofloxacin & 0 & 0 & 2 & 0 & 10 & 3 \\
\hline Gentamicin & 60 & 50 & 70 & 100 & 70 & 33 \\
\hline Tazocin & 90 & 50 & 6 & 40 & 70 & 0 \\
\hline Tetracycline & 60 & 20 & 16 & 40 & 80 & 8 \\
\hline $\begin{array}{l}\text { Trimethoprim- } \\
\text { Sulfame- } \\
\text { thoxazole }\end{array}$ & 40 & 0 & 14 & 40 & 30 & 20 \\
\hline Tigecycline & - & - & 91 & 50 & 100 & 67 \\
\hline Colistin & - & - & 10 & $\varnothing$ & $\varnothing$ & 11 \\
\hline Fosfomycin & 80 & 80 & 92 & 80 & 100 & 27 \\
\hline
\end{tabular}

\section{Discussion}

This is the first study that reports on the in vitro CZA activity against the most commonly encountered Gram-negative bacteria from Lebanon. An unanticipated high rates of resistance, as 
determined by MICs levels, were revealed against CR K. pneumoniae (65\%) and CR E. coli (60\%), despite the fact that this antimicrobial agent was only introduced in Lebanon in the second quarter of 2019 and procured at our medical center in July 2019. However, its activity remained uniformly high (100\%) against ESBL and MDR strains of both species, a similar finding to what was reported from regional and other countries [10-15].

Also in our study, the CZA activity by disk diffusion versus other antimicrobial agents routinely tested in our laboratory against CR E. coli and CR K. pneumoniae pathogens were compared. The findings revealed higher CZA activity compared to ciprofloxacin, piperacillin/tazobactam, tetracycline, SXT and colistin against both species. However, the activities of aminoglycosides and fosfomycin were higher than CZA among CR E. coli isolates but were closer to those among CR K. pneumoniae isolates (Table 2).

To make relevance, the activity of CZA against CR isolates in our study is compared to those reported internationally and regionally and indicated variable findings. Internationally for example, the $60 \%$ resistance rate detected among our CR E. coli isolates was lower than reported from China (71\%) [16]. However, the $65 \%$ resistant rate detected among our CR K. pneumoniae isolates was far higher than those reported from USA (0-21\%) [17, 11, 12], China (15\%) [16] and Brazil (3\%) [18].

Nevertheless, regionally no published data on integrate on species per se were reported, rather they were lump summed under Enterobacteriaceae. Among the latter, for example high rates of CZA resistance were reported from UAE (55\%) [23] and Arabian Peninsula (47\%) [24]. These were higher than rates reported from USA (range between 0.3\% and 3.6\%) [19, 20, 21] and China (25\%) [16, 22].

The mechanisms of resistance in Gram-negative bacteria generally fall under three main types: enzymatic resistance, expression of an alternative target or chemical modification of the antibiotic target, and expression of efflux pumps or changes in cell permeability [27]. The resistance to CZA was mainly ascribed to the enzymatic type of resistance, although combination with the two other mechanisms was also reported to further increase MIC levels [28]. In Enterobacteriaceae, the reported bacterial genes involved in CZA resistance included mutations among the following genes: KPC-2, KPC-3, CTX-M-14, CTX-M-15, SHV, AmpC, OXA-2, OXA48 and NDM [6]. In this context, studies indicated that the high CZA resistance rates against CRE were correlated with specific resistance genes existing in the pathogen. For example, in a global collection of $K$. pneumoniae isolates positive and negative for MBL gene, the resistance rate to CZA showed 98\% and $0.2 \%$, respectively [15]. Similar findings were also reported among tested isolates from Arabian Peninsula and Europe [24, 25]. A study from UAE also noted increased CZA resistance rates associated with specific genes as follows: $20 \%$ in OXA-48, $71 \%$ in NDM-1, and $95 \%$ in isolates containing dual genes OXA-48 and NDM-1 [23]. Moreover, a study done at a major cancer center in USA showed increased resistance to CZA among isolates harboring the NDM-1 gene [26].

At our Medical Center, the determination of genes involved in CZA resistance against the CR E. coli and CR K. pneumoniae isolates in the current study are being pursued. Unfortunately, this analysis was delayed due to the multifaceted problems inflicting Lebanon. However, earlier molecular studies from our institution revealed that $C R$ genes in both species were: bla-OXA-1, bla-CTXM-15, bla-TEM-1, bla-CMY-2, bla-OX-48 and NDM-1. In addition, E. coli isolates were found to harbor outer membrane porin encoding genes (OmpC and $\mathrm{OmpF}$ ) isolates while K. pnemoniae lacked these genes [29, 30, 31]. Whether these genes or newly emerged ones have been contributing to the CZA high rates of resistance encountered among the CRE isolates in our study remains to be determined. 


\section{Conclusion}

Although CZA was recently introduced in Lebanon, it was surprising and unexpected to note its low activity against CR E. coli and CR K. pneumoniae, thus posing a challenging issue concerning the treatment of carbapenem resistant Enterobacteriaceae. It is important to note that the findings in this study relate to investigation at a major tertiary care center and does not necessarily represent what is going on at the country level, as the latter would require a nationwide study. Minimizing the drug resistance necessitates pursuing investigations related to proper antimicrobial utilization in clinical practice and antimicrobial stewardship. Moreover, genotypic determination is needed to help explain the observed phenotypic resistance.

\section{Acknowledgements}

The support and technical assistance of the following Bacteriology staff is highly appreciated; Lina Itani, Rima Asmar, Rania Hammoud, Sarah Baydoun, Shafika Koubaitary, Dana Berjawi, Najwa Sayegh, Nadia Ayyash, and Hiba Halabi.

\section{References}

1. Tacconelli E, Pezzani MD. Public health burden of antimicrobial resistance in Europe. Lancet Infect Dis 2019; 19(1):4-6. doi: 10.1016/S1473-3099(18)30648-0. Epub 2018 Nov 5. PMID: 30409682.

2. Kaye KS, Pogue JM. Infections caused by resistant Gram-negative bacteria: Epidemiology and Management. Pharmacotherapy 2015; 35 (10): 949-962. doi: 10.1002/phar.1636. PMID: 26497481

3. Zasowski EJ, Rybak JM, Rybak MJ. The $\beta$-Lactams strike back: Ceftazidime-avibactam. Pharmacotherapy 2015; 35(8):755770. doi: 10.1002/phar.1622. PMID: 26289307; PMCID: PMC4545577.

4. Torres A, Rank D, Melnick D, Rekeda L, Chen X, Riccobene T et al. Randomized trial of ceftazidime-avibactam vs meropenem for treatment of hospital-acquired and ventilator-associated bacterial pneumonia (REPROVE): Analyses per US FDA-specified end points. Open Forum Infect Dis 2019 Apr 25; 6 (4):ofz149. doi: 10.1093/ofid/ofz149. PMID: 31041348; PMCID: PMC6483139.
5. Zhanel GG, Lawson CD, Adam H, Schweizer F, Zelenitsky $S$, Lagacé-Wiens PR et al. Ceftazidime-avibactam: a novel cephalosporin/ $\beta$-lactamase inhibitor combination. Drugs 2013; 73(2):159-177. doi: 10.1007/s40265-013-0013-7. PMID: 23371303.

6. Wang Y, Wang J, Wang R, Cai Y. Resistance to ceftazidimeavibactam and underlying mechanisms. J Glob Antimicrob Resist 2020; 22:18-27. doi: 10.1016/j.jgar. 2019.12.009. Epub 2019 Dec 19. PMID: 31863899.

7. Araj GF, Avedissian AZ, Itani LY, Obeid JA. Antimicrobial agents active against carbapenem-resistant Escherichia coli and Klebsiella pneumoniae isolates in Lebanon. J Infect Dev Ctries 2018; 12(3):164-170. doi: 10.3855/jidc.9729. PMID: 31829991.

8. Magiorakos AP, Srinivasan A, Carey RB, Carmeli Y, Falagas ME, Giske CG et al. Multidrug- resistant, extensively drug-resistant and pandrug-resistant bacteria: an international expert proposal for interim standard definitions for acquired resistance. Clin Microbiol Infect 2012; 18: 268-281

9. Araj GF, Ibrahim GY. Tigecycline in vitro activity against commonly encountered multidrug-resistant Gram- negative pathogens in a Middle Eastern country. Diagn Microbiol Infect Dis 2008; 62: 411- 415.

10. Flamm RK, Nichols WW, Sader HS, Farrell DJ, Jones RN. In vitro activity of ceftazidime/avibactam against Gram-negative pathogens isolated from pneumonia in hospitalized patients, including ventilated patients. Int J Antimicrob Agents 2016; 47(3):235-42. doi:

11. Gonzalez MD, McMullen AR, Wallace MA, Crotty MP, Ritchie DJ, Burnham CA. Susceptibility of ceftolozane-tazobactam and ceftazidime-avibactam against a collection of $\beta$-lactam-resistant Gram-negative bacteria. Ann Lab Med 2017; 37(2):174-176. doi: 10.3343/alm.2017.37.2.174. PMID: 28029009; PMCID: PMC5204000.

12. Hachem R, Reitzel R, Rolston K, Chaftari AM, Raad I. Antimicrobial activities of ceftazidime-avibactam and comparator agents against clinical bacteria isolated from patients with cancer. Antimicrob Agents Chemother 2017; 61(4):e02106-16. doi: 10.1128/AAC.02106-16. PMID: 28115350; PMCID: PMC5365661.

13. Yasir M, Ajlan AM, Shakil S, Jiman-Fatani AA, Almasaudi SB, Farman Met al. Molecular characterization, antimicrobial resistance and clinico-bioinformatics approaches to address the problem of extended-spectrum $\beta$-lactamase-producing Escherichia coli in western Saudi Arabia. Sci Rep 2018; 8(1):14847. doi: 10.1038/s41598-018-33093-8. PMID: 30287889; PMCID: PMC6172265. 
14. Hackel M, Kazmierczak KM, Hoban DJ, Biedenbach DJ, Bouchillon SK, de JongeBL et al. Assessment of the in vitro activity of ceftazidime-avibactam against multidrug-resistant Klebsiella spp. collected in the INFORM global surveillance study, 2012 to 2014. Antimicrob Agents Chemother 2016; 60(8):4677-83. doi: 10.1128/AAC.02841-15. PMID: 27216054; PMCID: PMC4958227.

15. Rossolini GM, Stone GG. Assessment of the in vitro activity of ceftazidime/avibactam against a global collection of multidrugresistant Klebsiella spp. from the INFORM surveillance programme (2015-2017). Int J Antimicrob Agents 2020; 56(3):106111. doi: 10.1016/j.ijantimicag.2020.106111. Epub 2020 Jul 25. PMID: 32721602.

16. Yin D, Wu S, Yang Y, Shi Q, Dong D, Zhu Det al. China antimicrobial surveillance network (CHINET) study group. Results from the China antimicrobial surveillance network (CHINET) in 2017 of the in vitro activities of ceftazidime-avibactam and ceftolozanetazobactam against clinical isolates of Enterobacteriaceae and Pseudomonasaeruginosa. Antimicrob Agents Chemother 2019; 63(4):e02431-18. doi: 10.1128/AAC.02431-18. PMID: 30617091; PMCID: PMC6437533.

17. Wilson WR, Kline EG, Jones CE, Morder KT, Mettus RT, Doi $Y$ et al. Effects of KPC variant and porin genotype on the in vitro activity of meropenem-vaborbactam against carbapenem resistant Enterobacteriaceae. Antimicrob Agents Chemother 2019; 63(3):e02048-18. doi: 10.1128/AAC.02048-18. PMID: 30617090; PMCID: PMC6395938.

18. Rossi F, Cury AP, Franco MRG, Testa R, Nichols WW. The in vitro activity of ceftazidime-avibactam against 417 Gram-negative bacilli collected in 2014 and 2015 at a teaching hospital in São Paulo, Brazil. Braz J Infect Dis 2017; 21(5):569-573. doi: 10.1016/j. bjid.2017.03.008. Epub 2017 Apr 20. PMID: 28435011.

19. Castanheira M, Mendes RE, Jones RN, Sader HS. Changes in the frequencies of $\beta$-lactamase genes among Enterobacteriaceae isolates in U.S. hospitals, 2012 to 2014: Activity of ceftazidimeavibactam tested against $\beta$-lactamase-producing isolates. Antimicrob Agents Chemother22; 60(8):4770-7. doi: 10.1128/ AAC.00540-16. PMID: 27216075; PMCID: PMC4958231.

20. Zhang Y, Kashikar A, Brown CA, Denys G, Bush K. Unusual Escherichiacoli PBP 3 insertion sequence identified from a collection of carbapenem-resistant Enterobacteriaceae tested in vitro with a combination of ceftazidime-, ceftaroline-, or aztreonam-avibactam. Antimicrob Agents Chemother 2017; 61(8):e00389-17. doi: 10.1128/AAC.00389-17. PMID: 28559260; PMCID: PMC5527577.
21. Sader HS, Castanheira M, Shortridge $D$, Mendes RE, Flamm RK. Antimicrobial activity of ceftazidime-avibactam tested against multidrug-resistant Enterobacteriaceae and Pseudomonasaeruginosa isolates from U.S. medical centers, 2013 to 2016. Antimicrob Agents Chemother 2017; 61(11):e01045-17. doi: 10.1128/AAC.01045-17. PMID: 28827415; PMCID: PMC5655084.

22. Zou C, Wei J, Shan B, Chen X, Wang D, Niu S. In vitro activity of ceftazidime-avibactam and aztreonam-avibactam against carbapenem-resistant Enterobacteriaceae isolates collected from three secondary hospitals in southwest China between 2018 and 2019. Infect Drug Resist 2020; 13:3563-3568. doi: 10.2147/IDR.S273989. PMID: 33116675; PMCID: PMC7567573.

23. Alatoom A, Elsayed H, Lawlor K, AbdelWareth L, El-Lababidi $\mathrm{R}$, Cardona Let al. Comparison of antimicrobial activity between ceftolozane-tazobactam and ceftazidime-avibactam against multidrug-resistant isolates of Escherichiacoli, Klebsiellapneumoniae, and Pseudomonasaeruginosa. Int J Infect Dis 2017; 62:39-43. doi: 10.1016/j.jijid.2017.06.007. Epub 2017 Jun 10. PMID: 28610832.

24. Sonnevend Á, Ghazawi A, Darwish D, Barathan G, Hashmey $R$, Ashraf $T$ et al. In vitro efficacy of ceftazidime-avibactam, aztreonam-avibactam and other rescue antibiotics against carbapenem-resistant Enterobacterales from the Arabian Peninsula. Int J Infect Dis 2020; 99:253-259. doi: 10.1016/j. ijid.2020.07.050. Epub 2020 Jul 29. PMID: 32738488.

25. Kazmierczak KM, de Jonge BLM, Stone GG, Sahm DF. In vitro activity of ceftazidime/avibactam against isolates of Enterobacteriaceae collected in European countries: INFORM global surveillance 2012-15. J Antimicrob Chemother 2018; 73(10):2782-2788. doi: 10.1093/jac/dky266. PMID: 30010894.

26. Aitken SL, Tarrand JJ, Deshpande LM, Tverdek FP, Jones AL, Shelburne SA et al. High rates of nonsusceptibility to ceftazidimeavibactam and identification of New Delhi metallo- $\beta$-lactamase production in Enterobacteriaceae bloodstream infections at a major cancer center. Clin Infect Dis 2016; 63(7):954-958. doi: 10.1093/cid/ciw398. Epub 2016 Jun 16. PMID: 27313265.

27. Schillaci D, Spanò $V$, Parrino B, Carbone A, Montalbano A, Barraja $P$ et al. Pharmaceutical approaches to target antibiotic resistance mechanisms. J Med Chem 2017; 60(20):8268-8297. doi: 10.1021/acs.jmedchem.7b00215. Epub 2017 Jun 20. PMID: 28594170.

28. Chalhoub H, Sáenz Y, Nichols WW, Tulkens PM, Van Bambeke F. Loss of activity of ceftazidime-avibactam due to MexABOprM efflux and overproduction of AmpCcephalosporinase in Pseudomonas aeruginosa isolated from patients suffering from cystic fibrosis. Int J Antimicrob Agents 2018; 52(5):697-701. doi: 10.1016/j.ijantimicag.2018.07.027. Epub 2018 Aug 3. PMID: 30081137. 
29. Baroud M, Dandache I, Araj GF, Wakim R, Kanj S, Kanafani $Z$ et al. Underlying mechanisms of carbapenem resistance in extended-spectrum $\beta$-lactamase-producing Klebsiella pneumoniae and Escherichia coli isolates at a tertiary care centre in Lebanon: role of OXA-48 and NDM-1 carbapenemases. Int J Antimicrob Agents. 2013 Jan; 41(1):75-9. doi: 10.1016/j. ijantimicag.2012.08.010. Epub 2012 Nov 9. PMID: 23142087.

30. Kissoyan KA, Araj GF, Matar GM. Prevalence of carbapenem resistance genes and corresponding MIC90 in Enterobacteriaceae at a tertiary care center in Lebanon. J Infect Dev Ctries 2018; 12(2.1):9S. doi: 10.3855/jidc.10097. PMID: 31804984

31. Dagher C, Salloum T, Alousi S, Arabaghian H, Araj GF, Tokajian S. Molecular characterization of carbapenem resistant Escherichia coli recovered from a tertiary hospital in Lebanon. PLoS One 2018; 13(9):e0203323. doi: 10.1371/journal.pone.0203323.

PMID: 30188911; PMCID: PMC6126819.

Publish in The International Arabic Journal of Antimicrobial Agents

The Journal is an open access peer-reviewed journal that publishes scientific papers about all aspects of antimicrobials. The journal will publish original research articles, reviews, brief reports and case reports dealing with basic and clinical antibacterial agents, antiviral, antiprotozoals, antituberculuous, antifungal and antihelminthes agents. All manuscripts must be prepared in English, and are subject to a rigorous and fair peer-review process. Accepted papers will immediately appear online. The journal aims to advance the knowledge, attitude and the research of chemotherapy in the Arabic world in cooperation with international, national scientific and public societies as well as research centers with similar aims and objectives. 